\title{
A MODEL FOR LEARNING TEAMWORK SKILLS
}

\author{
Virginie TESSIER \\ University of Montreal, Canada
}

\begin{abstract}
In an attempt to resolve some of the gaps associated with the pedagogical integration of teamwork in design curricula, this article seeks to share a model for learning teamwork skills. This model is the result of a multiple case study methodology based on the learning experiences of 22 design students. Data was collected during various team projects through questionnaires and interviews. In relation to the concept of the zone of proximal development, the coded data was organized by thematic categories and training levels to provide a practical tool to support teaching and assessment practices to support the learning of teamwork skills. The proposed model allows for a systemic understanding of the teamwork skills that should be acquired during design training to navigate with efficiency and confidence in the collective projects of design's community of practice. It motivates individual efforts toward collaborative initiatives in the hopes of more coherent teamwork processes.
\end{abstract}

Keywords: Design, teamwork, collaboration, process, project-based learning

\section{INTRODUCTION}

From the 1980 s to the mid-1990s, the design community started to recognise and value the social dimension of its processes. Bucciarelli [1] positioned design as a social practice, Cross and Cross [2] studied the distinctions of team design processes, and Goldschmidt [3] compared individual and collective practices. Since then, design has been more and more leaning toward shared and collective processes. As Goldschmidt [3] stated, the complexity of design projects led designers to be confronted with 'the need for multiple expertise and division of labour' (p. 189).

In recent years, professional designers have been invited to join projects initiated by many disciplines, ranging from medical products, ergonomic solutions, technological innovations, marketing initiatives, etc. In that sense, the designer has become a generalist that masters a creative process and analytical skills to converge to meaningful and inventive propositions.

Still, the integration of teamwork within design curricula around the world has not been the subject of consistent inquiry ensuring the coherence of its teaching, learning methods, and assessment practices. From our experience, we notice that teachers are proposing team projects as part of their classes or workshops, but most often without reflecting on the necessary skill set to gain performance for team projects or the optimal learning progression to achieve such performance. Among others, Tucker et al. [4] and Kleinsmann et al. [5] did start to draft typologies distributing teamwork experiences across typical design curricula. However, the lack of attention offered to assessment practices and methods to judge the performance of students or teams does not encourage the optimal integration of these frameworks within the pedagogical environment - which is composed of three equally important dimensions: teaching, learning, and assessing.

\subsection{Difficulties regarding the learning of complex teamwork skills}

Lack of structured approaches to implement teamwork within design curricula results in recurring challenges that are reported by various teachers and researchers. One recurrent challenge touches on the difficulty to bring students to collaborate. Collaboration is defined by specific characteristics that translate into a complex team dynamic, with high interdependence, shared comprehension, and common objective [6]. It has been noted that most students tend to work in teams according to less complex dynamics, such as cooperation or coordination, by distributing tasks and limiting exchanges $[7,8]$. However, although collaboration might ask for increased efforts from team members, important advantages should result in higher quality projects [9]. Such benefits have to be communicated to students, so they can perceive their value. Otherwise, they will not understand why it is worth investing 
time and effort. In the professional world, Stompff and Smulders [10] have noted that this tendency on the division of labour within teams leads to a lack of global vision resulting in fragmented solutions. In hopes of proposing paths for meaningful solutions to the identified gaps, this article will use the data collected through a multiple case study methodology. A detailed analysis will allow to make sense of the learning experiences of 22 design students during their team projects by creating links with the theoretical concept of the zone of proximal development, which understands learning as continuously ongoing and collectively influenced. Such an interpretation will allow us to propose a model to facilitate complex teamwork skills integration during design training. To conclude, the paper ends by identifying some of the benefits for design education and elaborates on the preparation of novices to navigate with confidence in the collective projects of design's community of practice.

\section{MULTIPLE CASE STUDY METHODOLOGY}

Wishing to gain a deeper understanding of the learning experiences of undergraduate students during their team projects, a multiple case study methodology was put together to access a complementary pool of experiences. Twenty-two undergraduate students were recruited to participate in the research from various design programs offered at the Faculty of environmental design of University of Montreal (Canada; industrial design, interior design, urban design). The participants were accepted in the research if they were working as a team on a workshop project during the period of data collection. For ethical reasons, all participation was determined on an individual basis (which results in sometimes having only one team member to comply with the research). Still, the participants of Group E worked in pairs and all teammates accepted to join the research. According to the pedagogical project, data collection varied between five to seven consecutive weeks. Participants were of various training levels and in strategically different learning situations to provide a scope of experiences. Figure 1 shares contextual information on each group.

\begin{tabular}{|c|c|c|c|c|c|}
\hline & Group A & Group B & Group C & Group D & Group E \\
\hline Discipline & Interior design & Urban design & Industrial design & Interior design & Industrial design \\
\hline Training year & First year & Third year (final) & Third year (final) & Fourth year (final) & Fourth year (final) \\
\hline Type of teamwork & Disciplinary & Disciplinary & Interdisciplinary & Interdisciplinary & Disciplinary \\
\hline $\begin{array}{l}\text { Data collection } \\
\text { (Length in weeks) }\end{array}$ & 6 weeks & 7 weeks & 5 weeks & 5 weeks & 6 weeks \\
\hline
\end{tabular}

Figure 1. Portrait of participants

All participants were asked to fill weekly questionnaires describing the state of their project, their personal or collective challenges, and their decision-making strategies. These questionnaires were designed to take less than 20 minutes to fill to encourage students to participate in the research. They offered information about the present events of the participating students. The content of the questionnaire was explained in more detail as part of a past EPDE conference [12].

The questionnaires were combined with a one-to-one interview at the end of the workshop projects. These interviews provided clarifications on the reported experiences described by the participants. They allowed the researcher to dig deeper into the challenges reported by the student and to gain a more accurate comprehension. Most of all, the interviews contributed to the understanding of the participant's vision of his or her experience. Interview questions were organized to bring the participant to gain perspective on his experience, develop its reflectivity regarding his team situation, and propose alternative ways to overcome or address the main or recurring challenges in the future.

This multiple case study methodology resulted in a mass of data from which sense needed to be made by finding patterns and creating meaning through data analysis, which is described next.

\section{DATA ANALYSIS}

All data from the questionnaires and the interview verbatim were transcribed in the coding software MAXQDA. A total of 97 codes emerged from that process. Cross-verification and code organization allowed us to combine or delete some of these codes, resulting in 33 codes describing the teamwork experiences of our 22 participants. Moreover, all codes were organized into five categories, 
corresponding to thematic groups around a shared topic. The codes and their five categories - interpreted as characteristics of teamwork learning experiences - are shared in the following Figure 2.

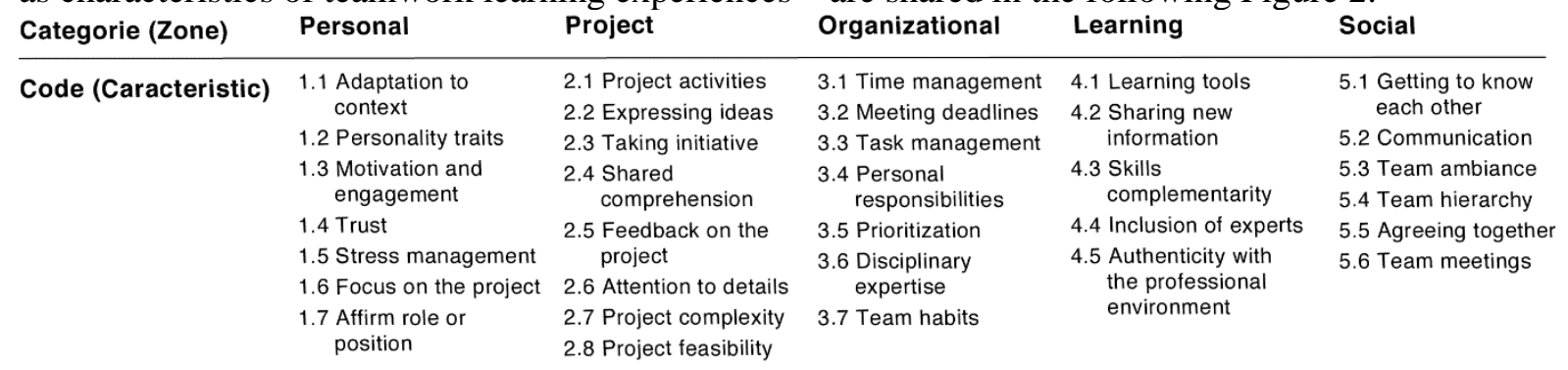

Figure 2. Characteristics of teamwork organized into 5 categories

All characteristics were analysed across the set of data, which allowed the identification of 81 analysis factors, increasing our specific and global comprehension. These factors allowed for pattern identification according to the levels of training, allowing the clear categorization of the new information. Unfortunately, the scope of this article does not allow us to provide more details of the descriptive factors. Still, a good example would be according to characteristic 2.2 - Expressing ideas that is associated with three factors: (a) Proposing ideas individually, (b) Combining ideas, (c) Fixation. These factors provide alternative stages in a team's effort to communicate possible insights according to a person's effort, a team's contribution or not being able to do either of these possibilities. Factor A was identified in all groups, Factor B only in some of them (Groups A, C, and E), and Factor C, only in group $\mathrm{E}$. These distinctions across groups of students show that not all students did achieve to combine their ideas as part of their teamwork experience. Moreover, investigating deeper into group E's difficulties translates the high complexity of their projects, resulting in a decline of some students' abilities to generate ideas.

Such an analysis was carried for all of the characteristics, to shed light on the similarities and distinctions of each group of students. The analysis also guided the identification of which characteristics and factors were predominant in the teamwork experiences of each group. Also, it underlined in particular which challenges were considered optimal for learning or too difficult concerning a certain learning context (for ex.: level of training or disciplinary/interdisciplinary). Such distinctions motivated the connection with the theoretical concept of the zone of proximal development.

\section{ZONE OF PROXIMAL DEVELOPMENT}

The zone of proximal development is a fundamental concept of the sociocultural perspective. This theoretical perspective was introduced by Vygotsky in the 1970s [11]. Through his study of children's development, Vygotsky came to understand cognitive growth as a continuous process according to which present abilities offer clues of one's future capacities.

The zone of proximal development is often illustrated as proposed in Figure 3. This representation shows the various stages that a learner encounters as part of its learning process. Still, the zone of proximal development is not entirely based on the sole learner, but also according to external support being provided to the learner as he develops more autonomy. Therefore, as shown in Figure 3, the central part of the model indicates all abilities that are mastered by an individual and that can be accomplished autonomously. The next section of the model translates what the learner can do with the help of a peer, teacher, or adult (often said to be a more capable peer). Finally, the external zone of the model identifies what the learner is not able to do either alone or with external help.

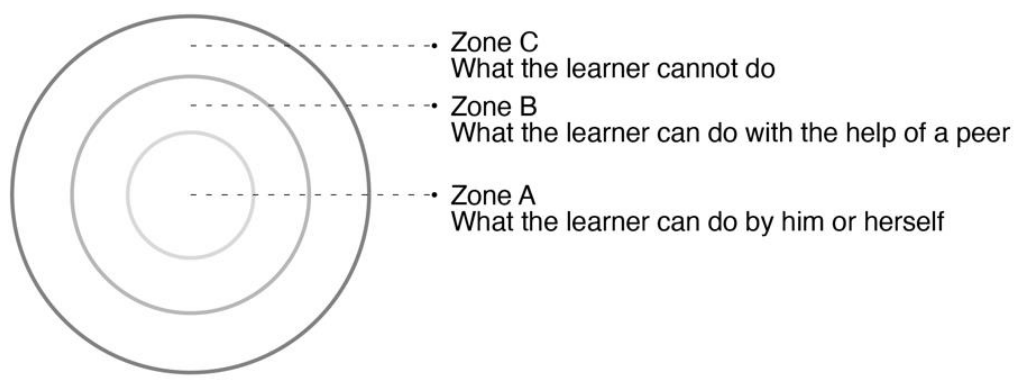

Figure 3. Zone of proximal development 
As part of the described learning process, social interactions are crucial for the learner to progress across the zones. Of course, travelling in the model is a cyclic process as the learner gains more autonomy for certain abilities, tasks, or knowledge. This program enables the learner to access more complex fields of knowledge, for which he or she needs external support.

\section{ZONE OF PROXIMAL DEVELOPMENT FOR TEAMWORK SKILLS MODEL}

The interactions that emerged between the data collected, the analytical interpretation, and our comprehension of the zone of proximal development guided the development of an integrative model. As shown in Figure 4 below, the model entitled 'zone of proximal development for learning teamwork skills' is strongly inspired by the initial zone of proximal development model. Still, some differences are noted. First, it is divided according to the five categories that emerged from our analysis process, creating zones of skills to master. Secondly, it is composed of four different circular levels. The core of the model represents the prerequisites that are requested by academic institutions. The next circle is concerned about first-year students, integrating a new environment based on high standards. As part of the personal zone, the participants corresponding to this stage showed they needed adaptive skills to adjust to the requirements of their undergraduate program. Similarly, the other zones also translate the need to acquire disciplinary-specific tools and skills to ensure a good progression throughout the following stages. Therefore, the project zone is set to understand and master the design process and the organizational zone seeks for the development of organizational skills to facilitate task division among team members. The learning zone is specific to mastering some of the basic tools of the designer such as drawing technics or software. Finally, the social zone asks to work on communicative skills, so students can share time with other individuals, recognize each other's forces and manage conflicts. These characteristics were mostly identified in Group A.

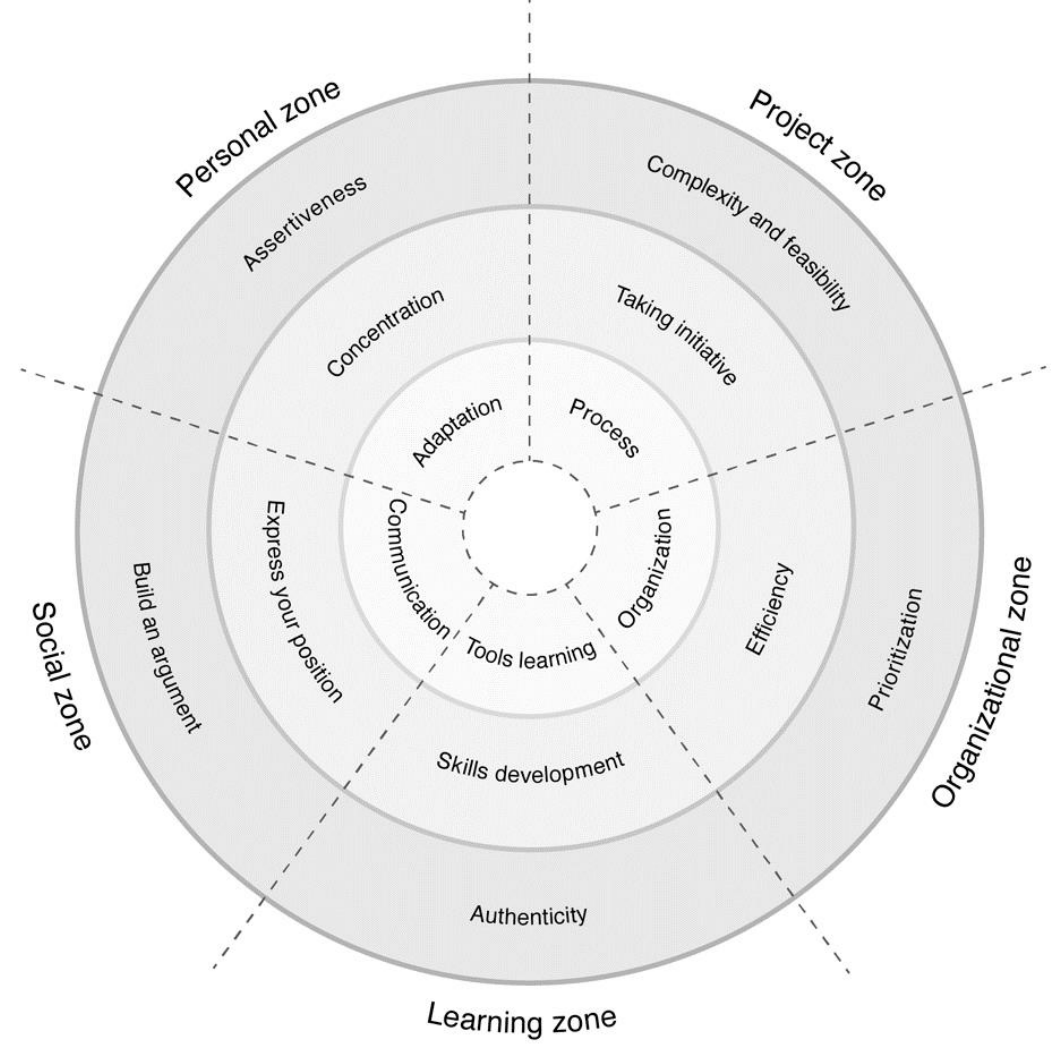

Figure 4. Zone of proximal development for learning teamwork skills model

The next circle is related to second- or third-year design students (depending on the curriculum). A different set of skills are identified in continuation with what was acquired in the previous level. Interpersonal relations tend to grow into friendship as students get to know each other: therefore, the personal zone is described in seeking concentration to stay focus and not disturbed by workshop stimuli. The next zone (project) is associated with students' initiatives to navigate more fluidly in the design process, take action and propose frame structuration. The organizational zone is concerned about 
efficiency to gain autonomy to formulate and accomplish tasks. The learning zone is related to skills development as a global improvement of disciplinary-specific and generic abilities. Lastly, the social zone corresponds to the development of the capacity to defend an opinion or position. As communication and relational skills should be practiced during various project experiences, one also as to be able to build its own perspective.

The last level, which leans to the professional world, corresponds to third- or fourth-year design students (last year of a training program). These students should be transitioning towards their future community of practice as they acquire a certain skill set in interactions with professionals. Both the first and last zone seek similar objectives in the construction of the professional identity of the student: affirmation of self and building an argument. The project zone and the learning zone both translate the authenticity of the proposed learning situations, the increased complexity of the project, and the feasibility of the proposed solution. The organizational zone proposes to facilitate prioritization during projects to meet the deadlines, comply with constraints, propose a realistic timeline, etc.

In brief, each level of the model corresponds to a specific range of training years but achieves coherence throughout a curriculum by progressing from an individual perspective through a more collaborative attitude. As stages are crossed by the students, more actors join in to contribute to the project with their specific expertise. In the first level, it is mostly the teacher that contributes as external help to the team project. At the second level, the peers or class colleagues are solicited for advice on ideas or the project. Finally, the third level seeks for more complex design projects and asks for the input of professionals according to specific domains of expertise to complement students' knowledge.

\subsection{Pedagogical uses of the model}

The proposed model has many benefits for design education:

- a framework for the coherent alignment of a pedagogical curriculum,

- a tool to structure the learning process,

- a perspective to develop reflective practice.

Biggs [13] raised the importance of planning a constructively aligned curriculum to provide a coherent structure to the learning process and increase students' investment in diverse categories of knowledge. Constructive alignment seeks to implement a structure between teaching, learning, and assessment practices of a class or workshop, but also, more globally, throughout the educational strategies and learning experiences of a curriculum. Such coherence supports deeper learning and a better understanding of the aimed objective.

In that sense, our model for learning teamwork skills offers a clear structure and sequence to gain autonomy with teamwork dynamics. The model proposes a progression from individual actions to collaborative initiatives by using the design project as its main motivation, guiding students from the centre of the model to its periphery. In that sense, the learner gains autonomy to accomplish teamwork characteristics in more complex contexts as he or she progresses in the model.

This model can be used to prepare teaching activities according to students' training level and to assess learning according to each characteristic's factors and whether the learner is lower or above the attended level. Moreover, the model can also be presented and explained to the students for them to take part in the judgement of their own performance. By using the model as a reference point among pedagogical actors, teachers, learners, or their teams can pinpoint the zone(s) where they feel less confident and see where they should be aiming. By understanding the global picture, design students would be empowered to reflect more deeply on their abilities and skills.

The proposed framework, based on the zone of proximal development, also supports the development of a reflective practice by perceiving the global picture and allowing discussions. During the interviews of our data collection, the participants were invited to discuss further the challenges they faced. The researcher tried to create a dialogue to deepen the perspective of the participant on its own experience. When successful, the participant was able to propose concrete ways to improve either their attitude, take actions or strengthen their team relationship and interactions. The capacity to find solutions based on their interpretation of the situation demonstrates an active cognitive process and a desire for change. As Argyris and Schön [14] demonstrated, professionals work according to their tacit knowledge, indicating that a large part of knowledge unfolds through imitation, observation, and interaction.

Building on this observation, it is possible to see how the model for learning teamwork skills could support the analysis of one's actions and behaviours, resulting in a more autonomous, constructive, and reflective practice. Offering common reference points to initiate conversation and reflection, the model 
should lead to a more thoughtful practice in the hopes of developing reflective habits in the behaviours of future professionals.

\section{CONCLUSIONS}

This article shared a research initiative that sought to understand teamwork experiences as design students live them. The analysis of qualitative data collected from 22 participants of various design programs and levels supported the development of a model based on the concept of zone of proximal development. This model, which is shared as the main contribution of this article, gathers 33 characteristics of teamwork design projects distributed in 5 zones (individual, project, organizational, learning, and social). These zones are subdivided into three levels corresponding to introductive, mid-, and advanced levels. The model can be used in support of all three fundamental activities of educational practice by facilitating teaching and pedagogical tasks, supporting learning, and offering guidelines for self-assessment of co-assessment. Such coherence within the pedagogical strategies of a training program can only be more positive for the learners by bringing them to see the global picture, understand the need for complex team dynamics, and offer clear stages to reach mastery of design as a social practice. Using the project as its main motivation, the model works around complementary skills for achieving teamwork coherence, as designers often take on the role of group facilitators in the projects they take part in [5].

\section{REFERENCES}

[1] Bucciarelli L. L. (1988). An Ethnographic perspective on engineering design. Design Studies, 9(3), 159-168.

[2] Cross N. and Cross A. C. (1995). Observations of teamwork and social processes in design. Design Studies, 16(2), 143-170.

[3] Goldschmidt G. (1995). The designer as a team of one. Design Studies, 16(2), 189-209.

[4] Tucker R., Abbasi N., Thorpe G., Ostwald M., Williams S. and Wallis, L. (2014). Enhancing and assessing group and team learning in architecture and related design contexts. Office for Learning and Teaching, Department of Education.

[5] Kleinsmann M., Deken F., Dong A., and Lauche K. (2012). Development of design collaborative skills. Journal of Engineering Design, 23(7), 485-506.

[6] Tessier V. (2020). Insights on collaborative design research: A scoping review. The Design Journal, 23(5), 655-676

[7] Zahedi M. and Heaton L. (2017). A model of framing in design teams. Design and Technology Education: An International Journal, 22(2), 8-25.

[8] Davies M. (2016). "Normal science" and the changing practices of design and design education. Visible Language, 50, 6-23.

[9] Tucker R. and Reynolds C. (2006). The impact of teaching models, group structures and assessment modes on cooperative learning in the student design studio. Journal for Education in the Built Environment, 1(2), 39-56.

[10] Stompff G. and Smulders F. (2013). Mirroring: The boundary spanning practice of designers. In C. de Bont, E. den Ouden, R. Schifferstein, F. Smulders, \& M. van der Voort (Eds.), Advanced Design Methods for Successful Innovation (p. 144-163). Design United.

[11] Vygotsky L. S. (1978). Mind in Society. Harvard University Press.

[12] Tessier V. and Zahedi M. (2019). Assessment of collaborative design: A sociocultural approach. In Proceedings of the 21 st International Conference on Engineering and Product Design Education. University of Strathclyde, Glasgow.

[13] Biggs J. (1996). Enhancing teaching through constructive alignment. Higher Education, 32(3), 347-364.

[14] Argyris C. and Schön D. A. (1977). Theory in practice: Increasing professional effectiveness. Jossey-Bass. 